appearance of the brain of very preterm infants and neurodevelopmental impairment at eight years. Dev Med Child Neurol Sept 1993; $\underline{35}$ :755-768). (Respond: Dr Simon C Roth, Dept Paediatrics, University College and Middlesex School of Medicine, The Rayne Institute, 5 University St, London WC1E 6JJ, England).

COMMENT. A normal ultrasound scan or a finding of uncomplicated periventricular hemorrhage in very preterm infants permits reassurance and the likelihood of a favorable developmental outcome. In contrast, infants with ventricular dilatation, hydrocephalus and cerebral atrophy have a poor prognosis. Learning difficulties experienced by some preterm infants may be caused by undetected hypoxic-ischemic injury.

Routine ultrasound screening in a multicenter study involving 15,151 pregnant women did not improve perinatal outcome when compared to the selective use of ultrasonography only for medical indications on the basis of clinical judgment (Ewigman BG et al. Effect of prenatal ultrasound screening on perinatal outcome. $\mathrm{N}$ Engl I Med Sept

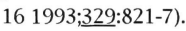

\title{
CEREBRAL HYPOPERFUSION IN MYOTONIC DYSTROPHY
}

Cerebral blood flow and perfusion measured by SPECT (Xenon and TcHMPAO), MRI findings, and neuropsychological function in 22 patients with myotonic dydtrophy (MD) were studied at the Departments of Neurology, Radiology, Psychiatry, and Pediatrics, Harbor-UCLA Medical Center, Torrance, CA. Results in 8 patients with maternal (mMD) inheritance were compared with those in 11 with paternal (pMD) inheritance, and in 10 normal controls. Patients with mMD had significantly lower scores on IQ tests and more extensive cerebral hypoperfusion when compared to those with pMD. Decreases in cerebral blood flow, most severe in frontal and temporoparietal association cortex, correlated strongly with lower scores on IQ tests. Patients with $\mathrm{mMD}$ had earlier onset of illness than the pMD group (means, 6.3 vs 32 yrs). Cerebral hypoperfusion patterns in the mMD patients were consistent with diffuse brain injury, whereas pMD patients showed only minor changes. (Chang L et al. Cerebral abnormalities in myotonic dystrophy. Cerebral blood flow, magnetic resonance imaging, and neuropsychological tests. Arch Neurol Sept 1993; $\underline{50}: 917-923)$. (Reprints: Dr Chang, Dept Neurology, Harbor-UCLA Medical Center, 1000 W Carson St, F-9, Torrance, CA 90509).

COMMENT. Mothers with myotonic dystrophy should be warned that their children would have a high probability of mental retardation. Hypoxic injury in utero is suggested as the likely explanation for the brain injury. Milder abnormalities in cerebral blood flow seen in the pMD group were attributed to possible sleep apnea, since many had hypersomnolence. 